\title{
Numerical Taxonomy of Bacteria - Some Published Data Re-examined
}

\author{
By D. W. GOODALL \\ C.S.I.R.O. Division of Mathematical Statistics, Western Australian Regional \\ Laboratory, Nedlands, Western Australia
}

(Received 21 June 1965)

SUMMARY

A probabilistic similarity index has been calculated from published data for the genera Chromobacterium and Mycobacterium, and for the Enterobacteriaceae. The resulting similarity matrices have been used as a basis for classification with significance tests.

The results show very good general agreement with the original analyses using simple Jaccard similarity indices, but the new index has proved rather more sensitive, and has enabled certain additional subdivisions to be made.

\section{INTRODUCTION}

Eight years ago Sneath $(\mathbf{1 9 5 7} a)$ published a first account of the use of computer methods for the classification of bacteria, and since that time a large number of papers have appeared in which the same or similar methods have been applied to other groups (see, for instance, Sneath's 1964 review of the subject).

Sneath used a very simple similarity index first introduced by Jaccard (1901) for the study of plant distribution and ecology, namely $100 a /(a+b+c)$, where $a$ was the number of positive two-value attributes shared by two strains, and $b$ and $c$ were the numbers of positive values shown by each strain separately, and not shared with the other. Most other workers since have used a modification of the same index (sometimes called the Simple Matching Coefficient) in which positive and negative records are treated alike, so that the expression becomes $[100(a+d)] /(a+b+c+d)$, where $d$ is the number of attributes absent from both strains; this inclusion of negative matches was agreed by Sneath (Sokal \& Sneath, 1963) to be a desirable improvement.

Once a matrix of similarity indices has been calculated, the question of how to use them in establishing a classification arises. The usual solution has been to group in a single taxon a set of OTUs (operational taxonomic units-a useful expression proposed by Sokal \& Sneath, 1963, to designate the entities whose classification is in question-individuals, strains, or low-rank taxa already recognized), each of which is linked to one or all others of the set by a similarity index exceeding a specified arbitrary limit. A lower limit is then set, and any new groups, accretions to the existing groups, or combinations of groups, recorded at this new level. Or the association of a new OTU with a group already recognized may be made to depend on the mean value of its similarity indices with all members of the group. 
Many workers using these methods of 'cluster analysis' have done so only with misgivings, because the clustering results could vary widely according to the particular arbitrary limits selected. Moreover, these methods did not lend themselves to significance tests, because the distribution of the indices under appropriate null hypotheses was not known.

'Natural' classification depends on correlation between the different classes of the values observed for a number of different attributes. If the question is raised whether a particular set of OTUs should be subdivided, an appropriate null hypothesis is consequently that the attribute values are assorted at random among them. If, on this null hypothesis, the probability of the observed degree of nonindependence in distribution of attribute values within the set is less than the chosen significance level, the null hypothesis is rejected and the set is subdivided. Such a significance test has not been developed in connexion with the usual similarity indices. Recently, however (Goodall, 1964, 1966), a new type of similarity index has been proposed which is the complement of the probability that two OTUs would have the observed (or a greater) degree of similarity if attribute values were assorted at random. In principle, this new index should lend itself to a classification controlled by a specified significance level.

A probabilistic similarity index of 0.95 , considered in isolation, is clearly just significant at the $5 \%$ level-there is a probability of exactly $0 \cdot 05$, on the null hypothesis, of obtaining a value as large or larger. But when a whole similarity matrix is under consideration its collective probability rather than that of a single index may be required. For instance, a similarity matrix for 20 individuals containing 190 indices would, on the null hypothesis of random assortment of attributes (implying a rectangular distribution of the indices) be expected to contain 19 indices $>0.90$ and nearly two $>0.99$; consequently a single pair of individuals with a similarity index of 0.99 is not to be regarded as constituting a cluster at the $1 \%$ significance level. For this, an index of $\mathbf{0 . 9 9 9 9 5}$ would be required, which is expected just once in 100 such matrices.

If, on the other hand, a set of 20 individuals included a subset of six, every pair of which had a similarity index of 70 or more, this subset must be regarded as constituting a cluster at a significance level of $1 \%, *$ for so large a subset linked by similarity indices all as high as this would be expected by chance less than once in 100 sets of twenty. This would be true even if none of the pairs individually had the index value required for significance.

The fact that significance of a larger cluster does not imply that smaller subsets within it are also significant clusters complicates the procedure for establishing a hierarchy of taxa. For this purpose several courses are open. One could, for example, apply a fixed significance level to the original similarity matrix, identify clusters of different sizes at this level, and regard the smaller clusters as subclasses at a lower hierarchic level within those larger clusters which include them. Or one could use a hierarchy of significance levels, smaller clusters identifiable at a higher significance level being included as subgroups within larger clusters at, a lower

\footnotetext{
* This, together with similar statements elsewhere in this paper, is based on an assumption of independence of the various similarity indices in the matrix-an assumption never fully valid in a practical situation. It will be shown, however, in a paper shortly to be published, that this is often an acceptable approximation.
} 
significance level. Neither of these procedures seems fully satisfactory, since within a significant cluster relations among similarity indices based on the whole population cannot be expected to follow expectations from a model of random assortment of attributes. On the other hand, if a new similarity matrix is calculated from the frequencies of attribute values within the cluster only (the indices in which will not necessarily be closely related to those between the same pairs in the original matrix), a new null hypothesis of random assortment of attributes within this more limited population may be set up and tested. Accordingly, this is the procedure which has been provisionally adopted. If significant clustering is detected, the largest significant clusters are separated, new similarity matrices calculated, and tests for clusters of subordinate rank performed. A detailed description of this procedure will be published shortly.

Some of the papers proposing bacterial classifications based on computer analysis have included full data on the attributes studied. It has seemed of interest to re-analyse these by the new methods proposed, and compare the results with those obtained by using simple similarity indices and arbitrary levels for distinguishing clusters. Three such sets of data have been re-analysed, with the results described below.

\section{RESULTS}

\section{Enterobacteriaceae}

In the study by Lysenko \& Sneath (1959) of fourteen species or species groups in the Enterobacteriaceae, thirty-one characters were included, in the main biochemical. Of these, twenty-five were described as either positive or negative (or as varying between strains in the group, in which case they were ignored), while the other six-sucrose, trehalose, $\mathrm{H}_{2} \mathrm{~S}$, citrate, glucose utilization, and glutamic acid-were recorded in more than one grade of positivity, and coded by the authors as two or more binary features. Since the probabilistic similarity index can take direct account of multi-value attributes, this binary coding was unnecessary, and the values were treated as ordered in the following sequence: prompt positive $>$ delayed positive $>$ late, irregular or mutated positive $>$ negative.

As Lysenko and Sneath said, the characters reported had been selected, so that these data could not show whether the groups are valid taxa. It may nevertheless be of interest to see whether these selected data support the accepted taxonomic relationships when probabilistic similarity indices are calculated.

The indices are reported in Table 1 . The large concentration of high values in the bottom right-hand corner of the table will be noted. The groups from 9 to 14 are all linked by similarity indices greater than $\mathbf{0 . 8 3}$, and only one of the fifteen is below $\mathbf{0} \cdot 95$. The chance that any such subset of groups could occur if the characters recorded had been randomly assorted among the groups is vanishingly small. The first eight bacteria also show a good deal of affinity, with nine (out of 28) similarity indices greater than $0 \cdot 9$, and 17 greater than $0 \cdot 7$, whereas between these two subsets (1-8 and 9-14) only 2 of the 48 similarity indices exceed $0 \cdot 5$.

These results, then, suggest a first division into two subsets, one including the groups from Escherichia to Serratia inclusive, the other the remainder including the two Proteus species. If now the process is repeated on these two subsets, the resulting similarity indices are those shown in Table 2. 
Table 1. Similarity indices for Enterobacteriaceae

Group no.

\begin{tabular}{|c|c|c|c|c|c|c|c|c|c|c|c|c|c|}
\hline Group name & 1 & 2 & 3 & 4 & 5 & 6 & 7 & 8 & 9 & 10 & 11 & 12 & 13 \\
\hline Escherichia & - & $\longrightarrow$ & - & - & 一 & 一 & - & 一 & - & - & 一 & - & 一 \\
\hline Salmonella & 0.924 & - & 一 & 一 & - & - & - & - & - & 一 & - & 一 & - \\
\hline Arizona & $0 \cdot 892$ & 0.996 & - & - & - & 一 & - & - & - & 一 & - & - & - \\
\hline Citrobacter & 0.872 & $0 \cdot 867$ & 0.920 & - & - & - & - & - & 一 & - & - & - & - \\
\hline Cloaca & 0.608 & $0 \cdot 622$ & $0 \cdot 769$ & $0 \cdot 793$ & - & - & - & - & - & - & - & - & - \\
\hline Klebsiella & 0.576 & $0 \cdot 411$ & $0 \cdot 742$ & $0 \cdot 744$ & 0.973 & 一 & - & - & 一 & 一 & - & - & - \\
\hline Hafnia & $0 \cdot 158$ & $0 \cdot 626$ & 0.901 & $0 \cdot 396$ & $0 \cdot 852$ & 0.981 & - & - & - & - & - & - & - \\
\hline Serratia & $0 \cdot 104$ & $0 \cdot 322$ & $0 \cdot 473$ & $0 \cdot 158$ & 0.932 & 0.968 & 0.938 & - & - & - & - & - & - \\
\hline Proteus vulgaris & $0 \cdot 136$ & $0 \cdot 034$ & $0 \cdot 153$ & 0.059 & 0.063 & $0 \cdot 040$ & $0 \cdot 052$ & $0 \cdot 825$ & - & - & 一 & - & - \\
\hline P. mirabilis & $0 \cdot 034$ & $0 \cdot 139$ & 0.381 & $0 \cdot 092$ & $0 \cdot 096$ & 0.001 & $0 \cdot 134$ & 0.758 & 1.000 & - & - & - & - \\
\hline Morganella & 0.421 & 0.026 & 0.014 & 0.004 & 0.001 & $0 \cdot 000$ & $0 \cdot 352$ & $0 \cdot 145$ & 0.997 & 0.999 & - & - & - \\
\hline Providencia & 0.373 & $0 \cdot 011$ & 0.002 & $0 \cdot 008$ & 0.006 & $0 \cdot 048$ & $0 \cdot 040$ & 0.537 & 0.974 & 0.961 & 1.000 & - & - \\
\hline Rettgerella & $0 \cdot 143$ & $0 \cdot 001$ & $0 \cdot 000$ & 0.004 & $0 \cdot 027$ & $0 \cdot 919$ & $0 \cdot 012$ & $0 \cdot 027$ & $0 \cdot 997$ & 0.832 & $0 \cdot 999$ & 1.000 & - \\
\hline Shigella type A1 & $0 \cdot 822$ & $0 \cdot \mathbf{4 4 8}$ & $0 \cdot 509$ & $0 \cdot 148$ & 0.017 & $0 \cdot 315$ & 0.012 & $0 \cdot 061$ & $0 \cdot 768$ & 0.947 & 0.999 & 0.999 & $\cdot 950$ \\
\hline
\end{tabular}

Table 3. Similarity indices between Mycobacterium strains

(a) $1,2,3,4,5,6,8,9,36,37$

(b) 13, 16, 17, 18, 19, 20, 22, 23, 38 35
24 24

(c) 6a, 11, 12, 14, 15, 21, 25 41 33

(d) $7,26,28$ 39

(e) $29,30,31,32$ 27 34 42
40

$\begin{array}{cccccccccccccc}\text { (a) } & (\text { b) } & \mathbf{3 5} & \mathbf{2 4} & 10 & \text { (c) } & 41 & 33 & \text { (d) } & 39 & \text { (e) } & 27 & 34 & 42 \\ 0.896 & - & - & - & - & - & - & - & - & - & - & - & - & - \\ 0.691 & 0.778 & - & - & - & - & - & - & - & - & - & - & - & - \\ 0.843 & 0.578 & - & - & - & - & - & - & - & - & - & - & - & - \\ 0.821 & 0.537 & 0.742 & - & - & - & - & - & - & - & - & - & - & - \\ 0.821 & 0.535 & 0.741 & 0.710 & - & - & - & - & - & - & - & - & - & - \\ 0.578 & 0.686 & 0.836 & 0.404 & 0.403 & 0.889 & - & - & - & - & - & - & - & - \\ 0.753 & 0.421 & 0.654 & 0.704 & 0.615 & 0.287 & - & - & - & - & - & - & - & - \\ 0.804 & 0.505 & 0.719 & 0.686 & 0.800 & 0.371 & 0.588 & - & - & - & - & - & - & - \\ 0.404 & 0.531 & 0.731 & 0.874 & 0.227 & 0.810 & 0.221 & 0.200 & 0.977 & - & - & - & - & - \\ 0.351 & 0.480 & 0.620 & 0.852 & 0.182 & 0.721 & 0.176 & 0.157 & 0.961 & - & - & - & - & - \\ 0.166 & 0.280 & 0.431 & 0.742 & 0.054 & 0.556 & 0.051 & 0.041 & 0.923 & 0.994 & 1.000 & - & - & - \\ 0.716 & 0.366 & 0.902 & 0.568 & 0.958 & 0.704 & 0.455 & 0.955 & 0.553 & 0.414 & 0.220 & - & - & - \\ 0.152 & 0.004 & 0.426 & 0.047 & 0.719 & 0.089 & 0.063 & 0.706 & 0.019 & 0.011 & 0.010 & 0.997 & - & - \\ 0.411 & 0.081 & 0.296 & 0.235 & 0.871 & 0.032 & 0.270 & 0.864 & 0.003 & 0.002 & 0.002 & 0.994 & 1.000 & - \\ 0.108 & 0.010 & 0.050 & 0.070 & 0.673 & 0.001 & 0.913 & 0.658 & 0.000 & 0.310 & 0.302 & 0.978 & 1.000 & 1.000\end{array}$


Within the eight groups forming the first subset, there is a strong suggestion that two smaller subsets (1-4 and 5-8) are more closely related. The twelve similarity indices within these subsets are all more than 0.66 , while only two of the sixteen indices between the two subsets exceed this value. The chance that a particular partition of eight species groups into two subsets of four should give twelve values all more than 0.66 is $(1-0.66)^{12}=2.4 \times 10^{-6}$, on the assumption of independence

Table 2. Similarity indices within subsets of Enterobacteriaceae

Group no....

Group name

1. Escherichia

2. Salmonella

3. Arizona

4. Citrobacter

5. Cloaca

6. Klebsiella

7. Hafnia

8. Serratia

Group no....

9. Proteus vulgaris

10. P. mirabilis

11. Morganella

12. Providencia

13. Rettgerella

14. Shigella type A1

Group no....

1. Escherichia

2. Salmonella

3. Arizona

4. Citrobacter

Group no....

5. Cloaca

6. Klebsiella

7. Hafnia

8. Serratia

Group no... .

11. Morganella

12. Providencia

13. Rettgerella

14. Shigella type A1
1

2

3

4

$\mathbf{5}$

6

7

of the similarity indices. Since there are seventy possible partitions of a set of eight into two subsets of four, the overall probability of the observed partition of similarity indices is less than $\mathbf{0 . 0 0 0 2}$, and the distinction between the two subsets must be adjudged highly significant.

Within the second subset (groups 9-14), the very high similarity index between the two Proteus species (more accurately, 0.999809) sets them apart from the rest, for the chance that at least one out of fifteen similarity indices derived from randomly assorted characters would be so high is only 0.003 .

The next stage in the analysis is accordingly to test whether the subsets 1-4, 5-8, and 11-14 show further internal relationships when they are treated separately. The results, also in Table 2 , give no firm grounds for a positive answer. The high similarities included are no higher, and no more numerous, than one could reason- 
ably expect in sampling from a rectangular distribution, nor do they give evidence of clustering.

In comparing the results of this analysis with that of Lysenko \& Sneath (1959), one first notes agreement in the close affinity of the two Proteus species, and in inclusion of Morganella, Providencia and Rettgerella in a broader group (Proteus sensu lato). Providencia and Rettgerella were placed very close together in both analyses. Shigella was placed in an isolated position by Lysenko \& Sneath, whereas here it is placed within the broader Proteus group; the difference arises because the Jaccard index used by Lysenko \& Sneath only takes presence of characters into account, whereas the index used here gives equal weight to their absence. The affinity of Shigella with Escherichia is however reflected in a fairly high similarity index $(0 \cdot 822)$, so that Shigella may to some extent form a bridge between the two main groups. Lysenko \& Sneath comment on the close relationship between Salmonella and Arizona, reflected here in similarity indices of $0.996,0.994$ and 0.842 in successive stages of the analysis. But the distinction between the first two subsets of four groups did not emerge from their treatment of the data.

\section{Mycobacterium}

Bojalil \& Cerbón (1961) studied 43 strains of Mycobacterium, recording 27 twovalue biochemical characters for each of them. There were five groups (including respectively $10,9,7,4$ and 3 strains) within each of which these characters were identical, while the other ten strains had sets of characters not shared with any other.

Probabilistic similarity indices for the whole set of data are recorded in Table 3. It will be noted that there is a concentration of high values in the bottom righthand corner of the table. The isolated strains $27,34,40$ and 42 form a subset (C) within which all similarity indices exceed 0.97 ; and groups (d) and (e), with strain 39, form another subset (B) with all indices greater than 0.92 . The occurrence of sets of similarity indices like this by random assortment of attributes is a highly unlikely event, so these may be regarded as groups of strains distinct from the remainder. Incidentally, all the other 31 strains (subset $A$ ) are linked by 465 similarity indices of which only 23 fall below $0 \cdot 5$, whereas between the three subsets thus recognized 281 out of 404 indices are less than 0.5 .

If these three subsets are separated and similarity indices recalculated within each subset, the results of Table 4 are obtained. Within subset $A$, six of the eight differing characters are recorded in single strains only-different in each case. The distribution of the other two characters so as to leave a group of seven strains (c) forming acid on neither mannitol nor salicin, a group of nine (b) forming it on salicin but not mannitol, and a third group of ten (a) forming it on both substrates is no reason for rejecting the null hypothesis of homogeneity.

Subset $B$ also need not be divided further, for the strains in this are distinguished by two attributes only (utilization of citrate, and formation of acid from fructose). Group (d) alone have the first attribute, and the chance that these three strains should also agree (as they do) in respect of the fructose response is $0 \cdot 143$-well above the usually accepted significance level. In subset $\mathrm{C}$, consisting of four strains, the highest similarity index recorded is likewise not such as to lead one to regard the subset as heterogeneous. 
Table 4. Similarity indices within subsets of Mycobacterium strains

Subset A

(a)

$\begin{array}{ll}\text { (a) } & 0.885 \\ \text { (b) } & 0 \cdot 544 \\ 35 & 0.771 \\ 24 & 0.765 \\ 10 & 0.769 \\ \text { (c) } & 0.271 \\ 41 & 0.750 \\ 33 & 0.638\end{array}$

(d) (b)

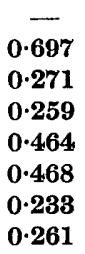

35

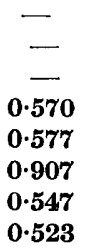

(e)

24
--
-
-
$\overline{-}$
0.567
0.035
0.750
0.375

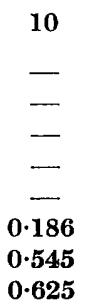

(c)

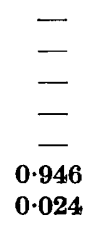

$0 \cdot 131$
Subset B

$\begin{array}{llrr}\text { (d) } & 0.983 & - & - \\ \text { 39 } & \mathbf{0 . 6 3 1} & - & - \\ \text { (e) } & 0 \cdot 158 & 0 \cdot 411 & \mathbf{0 . 8 2 2}\end{array}$
Subset C

$\begin{array}{rrrr} & 27 & 34 & 42 \\ 27 & - & - & - \\ 34 & 0 \cdot 762 & - & - \\ 42 & 0 \cdot 467 & 0 \cdot 980 & - \\ 40 & 0 \cdot 153 & 0 \cdot 494 & 0 \cdot 685\end{array}$

In this set of data, then, we are left with three homogeneous groups of strains, the variations within which may (as far as these data go) reasonably be ascribed to chance. These results are in accordance with the conclusions of Bojalil \& Cerbón (1961) themselves, using the Jaccard similarity index. They distinguished the same three groups of strains, the first and largest under the name of Mycobacterium fortuitum, while the other two were unnamed.

Table 5. Chromobacterium

Sneath's

feature nos.

$1-5$
$6-9$
$22-23$
$25-26$
$28,29,63$
$32-35$
$36-37$
$39-40$
$41-43$
$44-46$
$50-53$
$54-57$
$58-59$
$60-61$
$91-92$
$94-95$
$97-98$
$99-100$
$101-102$
$104-105$

Character

Length
Breadth
Presence of fat
Gelatinous consistency
Violacein production
Liquefaction in gelatin stab cultures
Digestion of Löffler serum slope
Colouration on potato slope
Heat resistance
Phenol resistance
Upper temperature limit for growth
Lower temperature limit for growth
Citrate utilization
Tolerance of NaCl
Nitrite destruction
Phosphatase test
Casein hydrolysis
Haemolysis of horse-blood agar
Egg-yolk reaction
Gluconate test

Number of ordered values

$\mathbf{4}$
$\mathbf{3}$
$\mathbf{3}$
$\mathbf{3}$
$\mathbf{4}$
$\mathbf{4}$
$\mathbf{3}$
$\mathbf{3}$
$\mathbf{3}$
$\mathbf{3}$
$\mathbf{4}$
$\mathbf{3}$
$\mathbf{3}$
$\mathbf{3}$
$\mathbf{3}$
$\mathbf{3}$
$\mathbf{3}$
$\mathbf{3}$
$\mathbf{3}$
$\mathbf{3}$

\section{Chromobacterium}

Finally we have as our largest set of data that published by Sneath (1957b), which he used in his original exposition of the application of similarity indices in bacterial classification. For 45 strains of Chromobacterium he recorded 70 characters, 
Table 6. Probabilistic similarity indices: Sneath's data for Chromobacterium

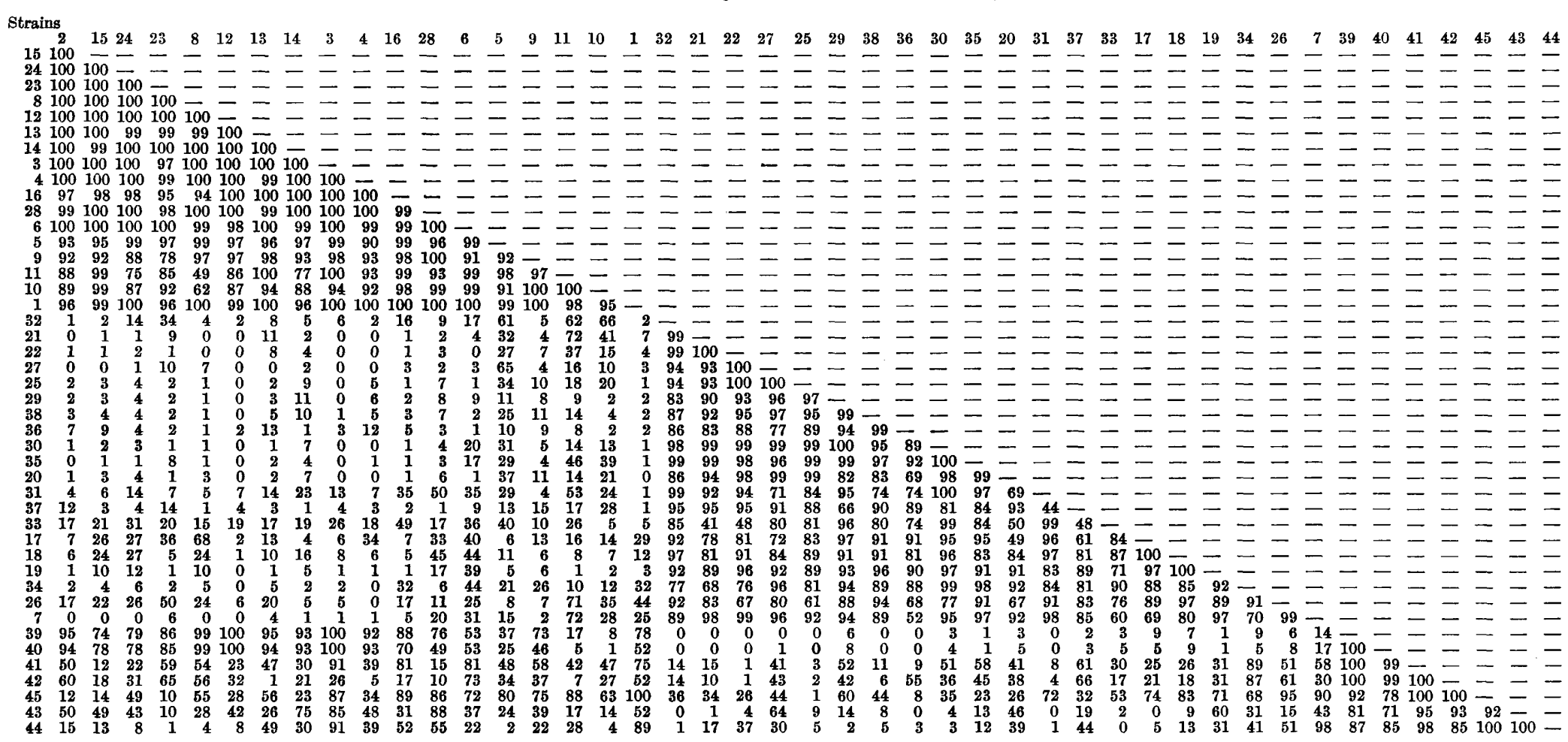


coded as 105 two-value features. For the present analysis, some of these characters have been recoded. The presence and arrangement of polar flagella (Sneath's features 16 and 18) were rescored as a three-value qualitative attribute, while twenty other characters, scored by Sneath as groups of binary features, were rescored each as a single attribute taking a number of ordered values, as shown in Table 5.

Table 7. Similarity indices for Chromobacterium after first subdivision

$$
\text { Group A }
$$

\begin{tabular}{|c|c|c|c|c|c|c|c|c|c|c|c|c|c|c|c|c|c|}
\hline Strain & 1 & 2 & 3 & 4 & 5 & 6 & 8 & 9 & 10 & 11 & 12 & 13 & 14 & 15 & 16 & 23 & 24 \\
\hline 2 & 3 & - & - & - & - & - & - & - & - & - & - & $\ldots$ & - & - & - & - & - \\
\hline $\mathbf{3}$ & 64 & 30 & 一 & - & - & - & - & - & - & - & - & - & - & - & - & - & $\ldots$ \\
\hline 4 & 21 & 74 & 100 & - & - & - & - & - & - & - & - & - & - & - & - & - & - \\
\hline 5 & 98 & 7 & 15 & 1 & - & - & - & - & - & - & - & - & - & - & - & - & - \\
\hline 6 & 86 & 59 & 9 & 23 & 98 & - & - & - & - & - & - & - & - & - & - & - & - \\
\hline 8 & 63 & 74 & 55 & 60 & 70 & 36 & - & - & - & - & - & $\ldots$ & - & - & - & - & - \\
\hline 9 & 99 & 23 & 21 & 6 & 94 & 55 & 46 & - & - & - & - & - & - & - & - & - & - \\
\hline 10 & 76 & 16 & 4 & 6 & 83 & 85 & 1 & 100 & - & - & 一 & - & - & - & - & $\ldots$ & - \\
\hline 11 & 71 & 14 & 27 & 9 & 98 & 80 & 0 & $\begin{array}{lll}98 & 1\end{array}$ & 100 & - & - & - & - & - & - & - & - \\
\hline 12 & 6 & 95 & 97 & 94 & 4 & 5 & 72 & 21 & 4 & 3 & - & - & - & - & - & - & - \\
\hline 13 & 85 & 67 & 46 & 6 & 26 & 65 & 23 & 74 & 37 & 93 & 66 & - & - & - & - & - & - \\
\hline 14 & 2 & 59 & 80 & 30 & 18 & 50 & 37 & 34 & 21 & 5 & 59 & 53 & - & - & - & - & - \\
\hline 15 & 16 & 91 & 43 & 58 & 8 & 53 & 78 & 21 & 60 & 57 & 74 & 69 & 28 & - & - & - & - \\
\hline 16 & 69 & 13 & 82 & 39 & 76 & 59 & $\mathbf{3}$ & 62 & 61 & 84 & 37 & 73 & 48 & 15 & - & - & - \\
\hline 23 & 31 & 85 & 2 & 23 & 59 & 691 & 100 & 13 & 32 & 16 & 60 & 33 & 62 & 67 & 8 & - & - \\
\hline 24 & 52 & 76 & 14 & 26 & 71 & 44 & 98 & 22 & 16 & 3 & 70 & 41 & 52 & 91 & 14 & 98 & - \\
\hline 28 & 75 & 36 & 23 & 63 & 43 & 77 & 61 & 97 & 90 & 39 & 32 & 23 & 86 & 34 & 40 & 23 & 30 \\
\hline & & & & & & & & & & & & & & & & & \\
\hline
\end{tabular}

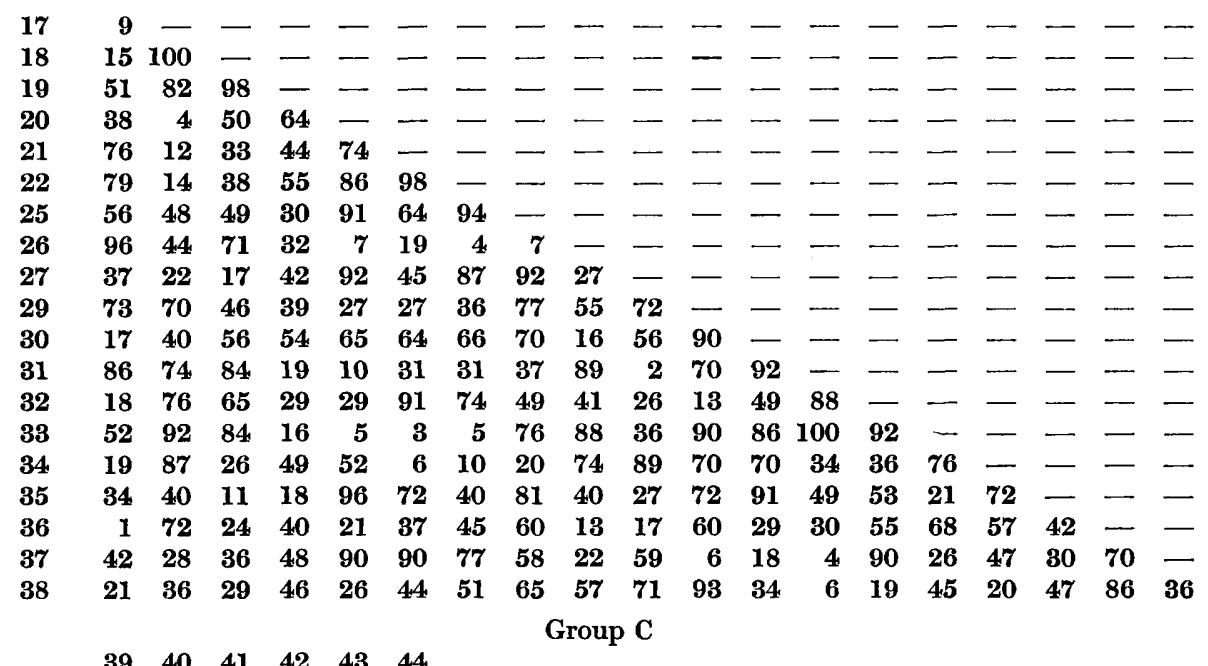


The similarity indices calculated from these recoded data are presented in Table 6. To facilitate comparison with Sneath's table 7, they are arranged in the same way and expressed in the same terms (as percentages rounded to the nearest unit).

Table 8. Similarity indices for Chromobacterium after second subdivision

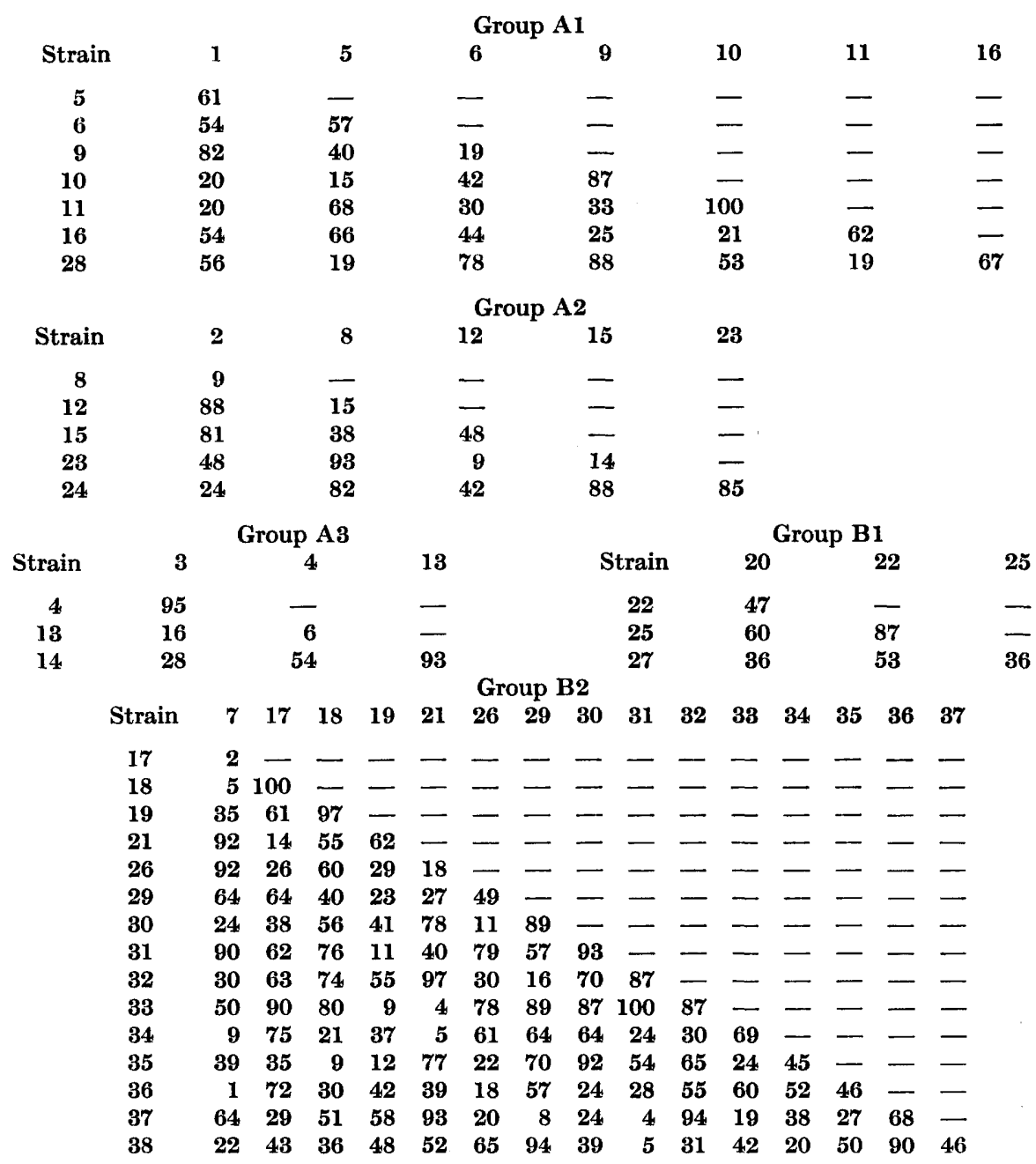

A first inspection of these figures suggests the following groupings:

A. Strains 1-6, 8-16, 23, 24 and 28 with 87 indices above $99 \%$, and only 5 less than $80 \%$.

B. Strains 7, 17-22, 25-27 and 29-38 with 22 indices above $99 \%$, and only 33 (out of 190) less than $80 \%$.

C. Strains 39-45 with 10 indices above $99 \%$, and only 4 less than $80 \%$.

Groups A and B here are identical with those separated by Sneath as mesophilic (a) and psychrophilic (b), while $\mathrm{C}$ includes all his five other small groups (c)-(g). 
The groups listed above were analysed separately, with the results shown in Table 7.

Within group A, two distinct subgroups may be recognized:

A1. Strains $1,5,6,9,10,11,16$ with all indices above $55 \%$. To these may perhaps be added no. 28-close to strains 1, 6, 9 and 10 but less so to the others. Further similarity analysis shows no significant clustering within A1 (see Table 8)-even the highest value (99.68) is such as might occur about once in ten such sets of data.

A2. Strains 2, 8, 12, 15, 23 and 24, with similarity indices all exceeding $59 \%$. Again, no significant groupings within A2 emerge (Table 8).

The remaining strains, $3,4,13$ and 14, may be placed together as a residue (group A3) after removing these more closely related groups. The index between the first two approaches significance at the $5 \%$ level, but apart from this these four may not be very closely related. Further analysis of this residue (Table 7) does not show any one pair as significantly similar. However, the indices for 3 and 4 and for 13 and 14 both exceed 0.93 , and the chance that four strains with random sets of attributes will be divisible into two pairs with similarities at this level is only $0 \cdot 015$. We may accordingly regard these as two small subgroups within $\mathbf{A}$, and designate them as A3 and A4.

We may note that the order in which the strains of group A are placed by Sneath accords exactly with the classification derived here, although he includes them all in his group (a). He places the strains of my A2 first, then A4, A3 and finally A1 as showing some affinity with group B. In a subsequent publication (Sneath, 1960) he described three 'indistinct subgroups' of the mesophilic strains, two of which were identical with my A2 and A3, while the third consisted of three strains $(9,10,11)$ included in my A1.

Within group B, only strains $20,22,25$ and 27 are significantly related, with a minimum index of $86 \%$ (Table 7 ); these are separated as $\mathrm{B1}$, while the remainder forms group B2. Further analysis of these subgroups (Table 8) does not reveal any further internal relationships. Sneath (1960) tentatively recognized three subgroups among the psychrophilic strains. Their existence as distinct taxonomic entities is not supported by the figures of Table 7, though three of the four strains in my $\mathrm{B} 1$ were included in the first of his subgroups.

Group C (Table 7) is clearly heterogeneous. The similarity indices between 39 and 40 , and between 43 and 44, both exceed $99.99999 \%$. Strains 41 and 42 are also closely related. Sneath likewise concluded that 39 and 40, 41 and 42 formed two distinct groups (c and d), but regarded 43 and 44 (for which his similarity index was only $63 \%$ ) as each representing a distinct group. The present analysis, however, leaves no doubt that, within the context of the strains he studied, the relationship of this pair was very close.

An example of overlapping is provided by strains 39 and 40 ; though included here in group $\mathbf{C}$ they also show some affinity with all of group $\mathbf{A}$ except strains 10 and 11. If, for instance, strains 39 and 40 had been combined with the six strains of group A2, the minimum similarity index within the group may be seen from Table 6 to be $74 \%$. Thus we may again regard strains 39 and 40 as bridging the gap between group $\mathbf{A}$ and the rest of group $\mathbf{C}$, and the strains may perhaps be placed in a sequence as follows: $(10,11),(1,5,6,9,16,28),(3,4,13,14),(2,8,12,15,23$, $24),(39,40),(41,42), 45,(43,44)$. 
Particularly when one is studying the structure of closely knit groups, it seems likely that overlapping and interlocking relations of this sort may be commoner than the discrete clusters assumed in the conventional taxonomic approach.

\section{DISCUSSION}

It is a tenet of most schools of numerical taxonomy that the various attributes considered should be given equal weight. At first glance, the use of a probabilistic similarity index might seem to offend this principle, since it gives differential weighting to attributes according to the commonness or rarity of the values observed. The objections to differential weighting, however, apply rather to a priori weights based on preconceptions as to the relative taxonomic importance of different attributes, and can have little force when the different weights are inherent in the data themselves. Similar procedures are countenanced by some of those advocating equal weighting. For instance, Sokal \& Sneath (1963), in relation to distance techniques, write of 'the automatic and quite appropriate weighting of mismatches by their magnitude'.

It must be understood that the similarity indices calculated here explicitly refer to a specified universe of discourse, and have no meaning in isolation. They are indeterminate for a particular pair of strains, until these strains are considered as two of a larger collection. This is also true according to one common convention, for the better-known indices. Many users (e.g. Hill, Tuiri, Gilardi \& Silvestri, 1961; Silvestri, 1962) feel that an attribute present in all the organisms considered contributes nothing to the similarity of any pair, and should be excluded in calculating the index. If this rule is followed consistently, the index will again be indeterminate when a pair is considered in isolation, and can only express the similarity of a pair in the context of a larger set.

When matrices of the simpler similarity indices are used for taxonomic purposes, a dendrogram (Sokal \& Sneath, 1963) is often prepared showing the minimum values of the indices at which successively larger groups are linked. The same could be done with a matrix of probabilistic similarity indices. This could at least ensure that within different phena recognized at any given level every pair of individuals was similar at a specified level of significance. But in doing this one would be throwing away a large part of the advantage of probabilistic treatment; the significance of a cluster depends on its size, as well as on the values of the indices and the size of the whole set under consideration. It is consequently not possible to use constant values of the index for the separation of phena without sacrificing a constant significance level. In the same way, a hierarchic treatment based only on the complete similarity matrix would not make the best use of the technique. Once the data have been found to include clusters the null hypothesis on which the probabilities are calculated is negatived, and new null hypotheses need to be set up if significance tests are to be valid.

The time required for the technique proposed here is obviously much longer than that needed for preparing dendrograms, for instance, based on matching coefficients. But the requirements are well within the powers of a modern electronic computer.

Apart from the obvious advantages that it facilitates special treatment of multivalue attributes and that it permits significance tests of hypotheses concerning the 
classifications proposed, the similarity index used here is shown to be more sensitive in enabling smaller groupings to be distinguished. Both in the data for Chromobacterium and for the Enterobacteriaceae subdivisions have been recognized additional to those of the original authors. By and large, though, the results in the two analyses are remarkably similar, and the results obtained with the Jaccard similarity index have in general been confirmed. It has often been suggested that, provided large numbers of unrelated attributes are taken into account, the results of different classificatory procedures may be expected to approach one another. The results reported here provide further support for this view.

I am indebted to Dr C. A. Parker and Dr P. H. A. Sneath for helpful comments. and to Mr D. W. G. Moore of the University of Western Australia and Dr G. N, Lance of the C.S.I.R.O. Computing Research Section in Canberra for computer facilities.

\section{REFERENCES}

Bojalil, L. F. \& Cerbón, J. (1961). Taxonomic analysis of nonpigmented, rapidly growing Mycobacteria. J. Bact. 81, 338.

Goodall, D. W. (1964). A probabilistic similarity index. Nature, Lond. 203, 1098.

Goodall, D. W. (1966). A new similarity index based on probability. Biometrics (in the Press).

Hill, L. R., TurRi, M., Gilardi, E. \& Silvestri, L. G. (1961). Quantitative methods in the systematics of Actinomycetales. II. G. Microbiol. 9, 56.

JACCARD, P. (1901). Distribution de la flore alpine dans le Bassin des Dranses et dans quelques régions voisines. Bull. Soc. Sci. nat. vaud. 37, 241.

Lysenko, O. \& SNeAth, P. H. A. (1959). The use of models in bacterial classification. J. gen. Microbiol. 20, 284.

Silvestri, L. G. (1962). Computer correlation of microrganisms. In Data Acquisition and Processing in Biology and Medicine. Proceedings of Conference in New York, 1962, p. 43. Oxford: Pergamon Press.

Sneath, P. H. A. (1957 a). Some thoughts on bacterial classification. J. gen. Microbiol. 17,184 .

Sneath, P. H. A. (1957b). The application of computers to taxonomy. J.gen. Microbiol. $17,201$.

SNEATh, P. H. A. (1960). A study of the bacterial genus Chromobacterium. Iowa St. J. Sci. 34, 243.

SNeath, P. H. A. (1964). New approaches to bacterial taxonomy: use of computers. A. Rev. Microbiol. 18, 335.

Sokal, R. R. \& Sneath, P. H. A. (1963). Principles of Numerical Taxonomy. San Francisco and London: W. H. Freeman \& Co. 